\title{
Efecto del pH ácido y de la desaireación del medio en el comportamiento frente a la corrosión de la MA 956 preoxidada
}

\author{
M.C.GARCÍA-ALONSO ${ }^{1}$,M.L.ESCUDERO ${ }^{1}$, J.L.GONZÁLEZ-CARRASCO ${ }^{1}$,C.CLEMENTE ${ }^{2}$ \\ ${ }^{1}$ Centro Nacional de Investigaciones Metalúrgicas, CENIM, CSIC, \\ 2 Facultad de Medicina de la Universidad de Alcala de Henares.
}

\begin{abstract}
Además del contenido en iones cloruro que ya hacen del medio fisiológico un entorno corrosivo, en la vida de un implante se producen situaciones transitorias que pueden provocar cambios locales del medio en torno al implante. En los primeros días de la implantación de un biomaterial es relativamente frecuente que se produzcan hematomas, con bajada en el valor del pH, inflamación e incluso infección. Estas situaciones químicas cambiantes, aunque en general son transitorias pueden modificar drásticamente el comportamiento a corrosión de un biomaterial y por tanto afectar negativamente a la vida en servicio de las prótesis.

En este trabajo se presenta el comportamiento a corrosión de la superaleación MA 956 con capa de alúmina, en suero fisiológico, en condiciones de bajo pH y desaireación para simular la posibilidad de hematomas, inflamación e infección de los tejidos que rodean la prótesis, dado que esta superaleación está propuesta como nuevo biomaterial.
\end{abstract}

Palabras clave: corrosión, pH, MA 956, alúmina, desaireación

Influence of an acid $\mathrm{pH}$ and desoxygenation on the in vitro corrosion behaviour of preoxidised MA 956

The lifetime of implants is affected by the aggressivity of the physiological medium, which mainly results from the relatively high concentration of ion chlorides. There are, however, other transient situations that may enhance the aggressivity of the environment in the adjacent zones to the implant. For example, during the first days of the implantation is rather frequent to get bruises, inflammation, and even infections. These modifications could dramatically influence the corrosion behaviour and decrease the life expectancy of the implant. In this work the corrosion behaviour of the MA 956, which has been proposed as a new biomaterial, is investigated under experimental conditions of low $\mathrm{pH}$ and deareation, which simulate the most typical medical complications in service.

Key words : corrosion, $p H, M A 956$, alumina, deaeration

\section{INTRODUCCIÓN}

La resistencia a la corrosión de los biomateriales que se implantan en el cuerpo humano y su biocompatibilidad son dos factores íntimamente relacionados debido a que generalmente los productos procedentes de la corrosión de estos implantes tienen efectos adversos sobre el organismo. En implantes osteoarticulares, por ejemplo los procesos biológicos necesarios para la formación de la matriz ósea o cartilaginosa, son especialmente susceptibles a interrumpirse ante la presencia de contaminantes tóxicos (1). Los materiales metálicos desnudos o recubiertos por cerámicas como alúmina o hidroxiapatita deben poseer la capacidad de resistir el ataque del medio fisiológico que los rodea $(2,3)$. Por otro lado, las condiciones del entorno corrosivo son decisivas en el comportamiento frente a la corrosión de cualquier material utilizado como implante. En condiciones normales, los fluidos extracelulares del cuerpo que constituyen el medio agresivo se pueden considerar como una disolución acuosa de oxígeno y diversas sales como $\mathrm{NaCl}, \mathrm{MgCl}, \mathrm{KCl}$, glucosa, etc., con un $\mathrm{pH}=7.4$ aproximadamente. Se trata pues de un medio electrolítico que contiene iones cloruro, que conduce electrones, lo que facilita que puedan tener lugar fenómenos electroquímicos de corrosión e hidrólisis (3) . Además del contenido en iones cloruro que ya hacen del medio fisiológico un entorno corrosivo, en la vida de un implante se producen situaciones transitorias que pueden provocar cambios locales del medio en torno al implante. En los primeros días de la implantación de un biomaterial es relativamente frecuente, que debido al propio trauma quirúrgico se produzcan hematomas, inflamación e incluso infección. Esto hace que el $\mathrm{pH}$ pueda disminuir localmente hasta valores ácidos de 5.6 ó 5.3 y que la oxigenación de los tejidos adyacentes a la prótesis no sea la adecuada $(4,5)$. Este entorno químico que cambia, temporalmente, en zonas muy determinadas, puede modificar drásticamente el comportamiento a corrosión de un biomaterial y por tanto afectar negativamente a la vida en servicio de una prótesis.

El objetivo de este trabajo es estudiar el comportamiento a corrosión de la superaleación MA 956 con capa de alúmina, en suero fisiológico, tanto en condiciones normales como frente a bajadas de $\mathrm{pH}$ y de oxígeno para simular la posibilidad de hematomas, inflamación e infección de los tejidos que rodean al implante.

\section{PROCEDIMIENTO EXPERIMENTAL}

La MA 956 es una superaleación ferrítica procesada vía pulvimetalúrgica mediante aleado mecánico (MA) y tratamientos termomecánicos. Su composición química es Fe-20Cr5Al-0,5Ti-0.5 $\mathrm{Y}_{2} \mathrm{O}_{3}$ (\% en masa). Esta aleación sometida a un 
tratamiento de oxidación, en un horno, a una temperatura de $1100^{\circ} \mathrm{C}$ en atmósfera de aire genera una capa superficial de $\alpha-\mathrm{Al}_{2} \mathrm{O}_{3}$, de espesor variable en función del tiempo de tratamiento.

La resistencia a la corrosión fue evaluada mediante la técnica de espectroscopía de impedancia electroquímica (EIE). En esta técnica se aplica una señal sinusoidal de $10 \mathrm{mV}$ en amplitud sobre el electrodo de trabajo, en un rango de frecuencia que varía desde 64000 a $0.001 \mathrm{~Hz}$. Como electrodo de referencia fue usado el electrodo de calomelano, saturado y como contraelectrodo un electrodo de platino.

El medio electrolítico empleado fue la solución de Hank cuya composición queda reflejada en la tabla I. Dicha solución simula los fluidos extracelulares del cuerpo humano, con un $\mathrm{pH}$ de 7.4 , y a una temperatura de $37^{\circ} \mathrm{C}$. Esta solución fue acidificada con $\mathrm{HCl}$ hasta alcanzar un $\mathrm{pH}$ ácido $(\mathrm{pH}=5.4)$ y sometida a una corriente de $\mathrm{N}_{2}$ con el fin de desairear el medio para simular condiciones que se producen in vivo de hematoma, inflamación e infección.

\section{RESULTADOS Y DISCUSIÓN}

La figura 1 muestra, a modo de ejemplo, el diagrama de Bode del módulo de impedancia y del ángulo de fase frente a la frecuencia, en condiciones normales de oxigenación a $\mathrm{pH}=7.4$, para muestras preoxidadas de MA 956. Con fines comparativos se han añadido los resultados para la misma aleación sin tratar, es decir sin capa. En el diagrama de la fig. 1 a se puede observar una recta de pendiente -1 , y unos valores del ángulo de fase, fig. 1 b, que se sitúan en torno a -90, en todo el intervalo de frecuencias estudiado, con ausencia de un rellano, a bajas frecuencias que evidencie que existe una resistencia asociada a los microdefectos de la capa. Este tipo de respuesta indica que la señal de potencial sinusoidal aplicada se invierte en cargar y descargar el condensador de la capa de alúmina, sin conducir prácticamente ninguna señal eléctrica a través de los microdefectos de la capa. Estos resultados están ampliamente refrendados en trabajos anteriores (6-8). En este trabajo se pretendía evaluar el comportamiento de esta aleación preoxidada en solución de Hank en condiciones de desaireación y bajo $\mathrm{pH}(\mathrm{pH}=5.4)$ con el fin de simular posibles hematomas, inflamación e infección de los tejidos; situaciones que se pueden producir in vivo en torno a la aleación.

En la figura 2 aparece la evolución de los diagramas de Bode a lo largo del tiempo de ensayo, de muestras preoxidadas 3 horas a $1100^{\circ} \mathrm{C}$ en solución de Hank a pH=5.4. En el diagrama de Bode del módulo de impedancia frente a la frecuencia, fig. 2a, los valores del módulo de impedancia a la frecuencia más baja son ligeramente menores a medida que aumenta el tiempo de ensayo. El diagrama de Bode, fig. $2 b$, del ángulo de fase frente a la frecuencia presenta valores del ángulo próximos a $-90^{\circ}$, en todo el rango de frecuencias ensayado, con un pequeño mínimo a $-80^{\circ}$ a una frecuencia de aproximadamente $1 \mathrm{kHz}$. En esta gráfica se puede observar igualmente una ligerísima tendencia a disminuir los valores de ángulo de fase a medida que aumenta el tiempo de ensayo. Estas ligeras desviaciones del módulo de la impedancia y del angulo de fase podrían estar asociadas con un proceso de disolución muy lento pero paulatino de la capa de alúmina a estos valores de $\mathrm{pH}$. Con el fin de comprobar si existe o no proceso de disolución, de la capa de alúmina a $\mathrm{pH}$ ácidos se hizo un seguimiento de los valores de la capacidad con el tiempo de ensayo, para muestras sometidas a solución de Hank de $\mathrm{pH}=5.4$. La figura 3 muestra la evolución de esta
Table I. Composición química de la SOlución de HanK en G/L

\begin{tabular}{|c|c|c|c|c|c|c|c|c|}
\hline $\mathrm{NaCl}$ & $\mathrm{CaCl}_{2}$ & $\mathrm{KCl}$ & $\mathrm{NaHCO}_{3}$ & Glucosa & $\begin{array}{c}\mathrm{MgCl}_{2} \cdot \\
6 \mathrm{H}_{2} \mathrm{O}\end{array}$ & $\begin{array}{c}\mathrm{Na}_{2} \mathrm{HPO}_{4} 2 \mathrm{H}_{2} \mathrm{O} \\
\mathrm{KH}_{2} \mathrm{PO}_{4}\end{array}$ & $\begin{array}{c}\mathrm{MgSO}_{4} \\
7 \mathrm{H}_{2} \mathrm{O}\end{array}$ \\
\hline 8 & 0.14 & 0.4 & 0.35 & 1 & 0.1 & 0.06 & 0.06 & 0.06 \\
\hline
\end{tabular}

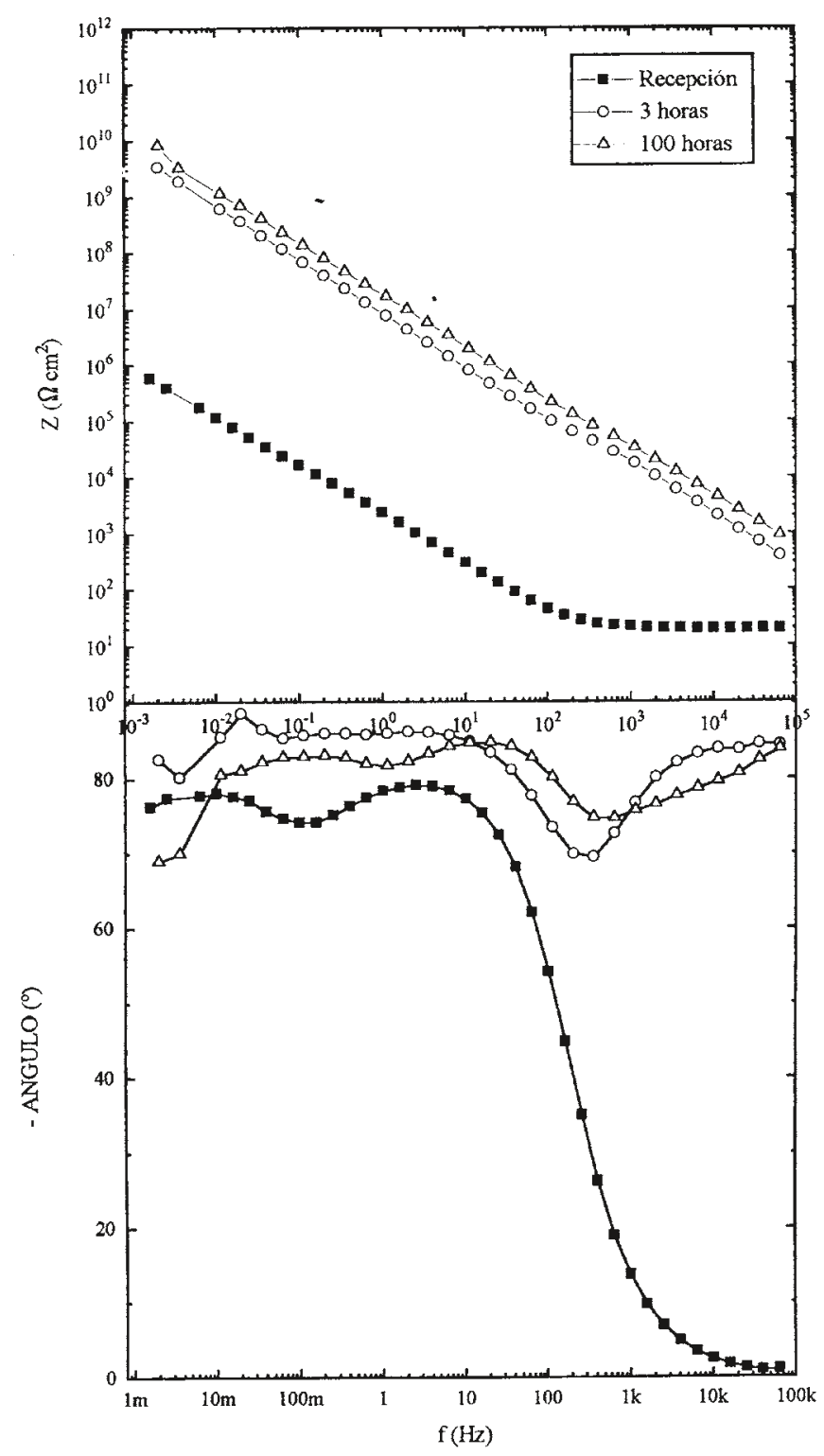

Fig.1 .- Diagramas de Bode obtenidos de la aleación MA 956 preoxidada a $1100^{\circ} \mathrm{C}$, y sin tratar, para muestras en solución de Hank aireada de $\mathrm{pH}=7.4$

capacidad con el tiempo de ensayo. Si comparamos esta evolución con la de muestras tratadas 3 y 100 horas y sumergidas en disolucíon de Hank de $\mathrm{pH}=7.4$, fig. 4, podemos observar una evolución completamente paralela. En las primeras 24 horas el valor de la capacidad aumenta ligeramente y después se estabiliza con el tiempo con independencia del valor del $\mathrm{pH}$ del medio. Teniendo en cuenta el diagrama de Pourbaix (9) la $\alpha$-alumina permanece estable a $\mathrm{pH}$ neutros, por lo que es muy poco probable que sea atacada. También se ha corroborado en investigaciones anteriores que los diagramas de impedancia no evolucionan significativamente para tiempos 


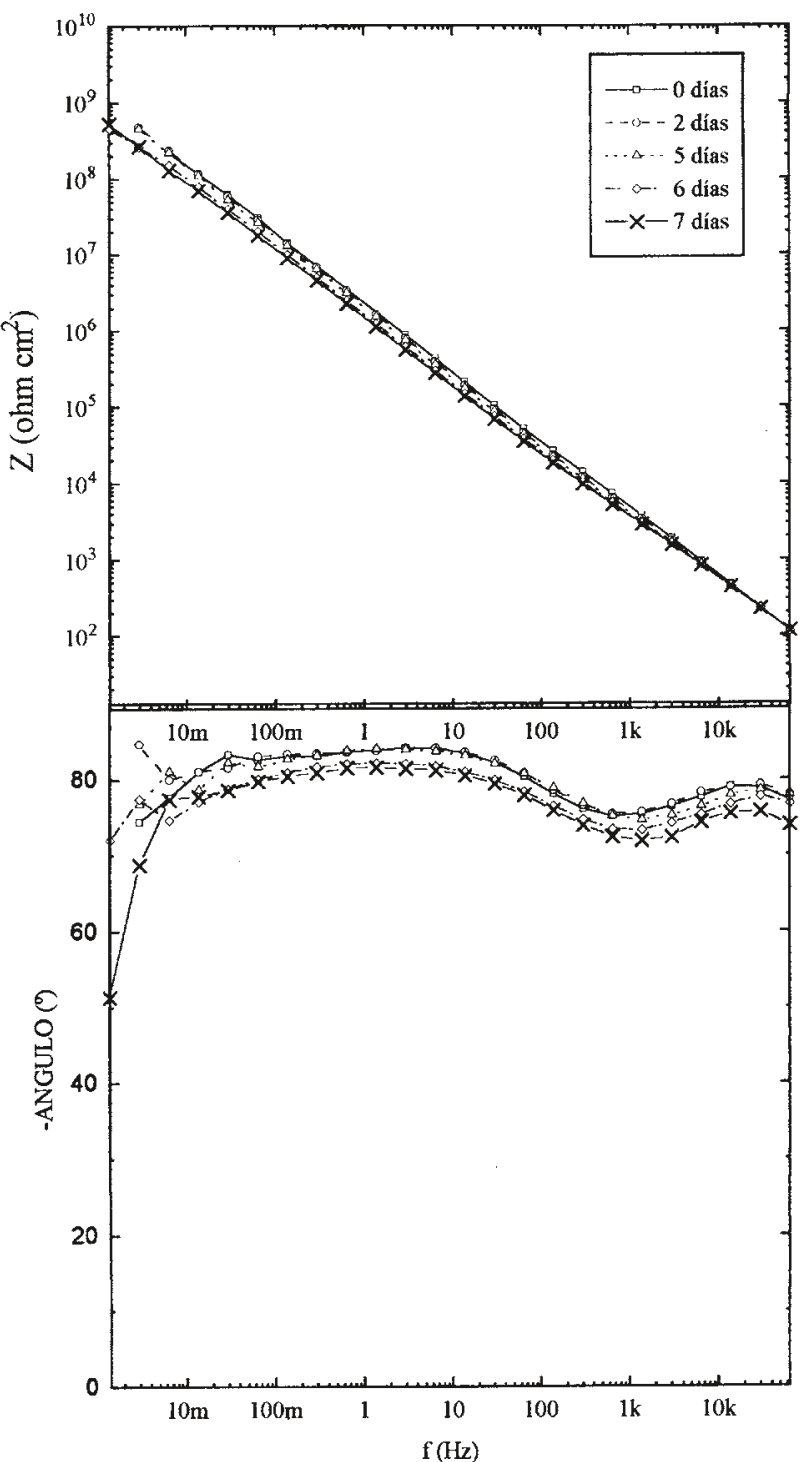

Fig.2.- Evolución de los diagramas de Bode a lo largo del tiempo de ensayo de muestras preoxidadas 3 horas a $1100^{\circ} \mathrm{C}$ en solución de Hank a $\mathrm{pH}=5.4$.

de ensayo largos, incluso despues de 9 meses (6). Por tanto el aumento de capacidad inicial más que atribuirlo a un proceso de disolución de la capa de alúmina en $\mathrm{pH}$ ácidos parece estar asociado con un ligero aumento en la constante dieléctrica del recubrimiento de alumina (10). Es muy probable que la disolución de Hank penetre lentamente en la capa de alúmina ocupando los pequeños microdefectos existentes en ella y; teniendo en cuenta que el agua tiene una constante dieléctrica de 80 frente a 8.3 de la alúmina, se puede aumentar ligeramente el valor de la capacidad del recubrimiento al principio de los ensayos. La evolución con el tiempo de los diagramas pone de manifiesto que esta impregnación del medio corrosivo en los microdefectos no conduce en ningún momento a la derivación de un proceso corrosivo. De lo que se deduce que la capa de alúmina de la aleación preoxidada MA 956 es muy

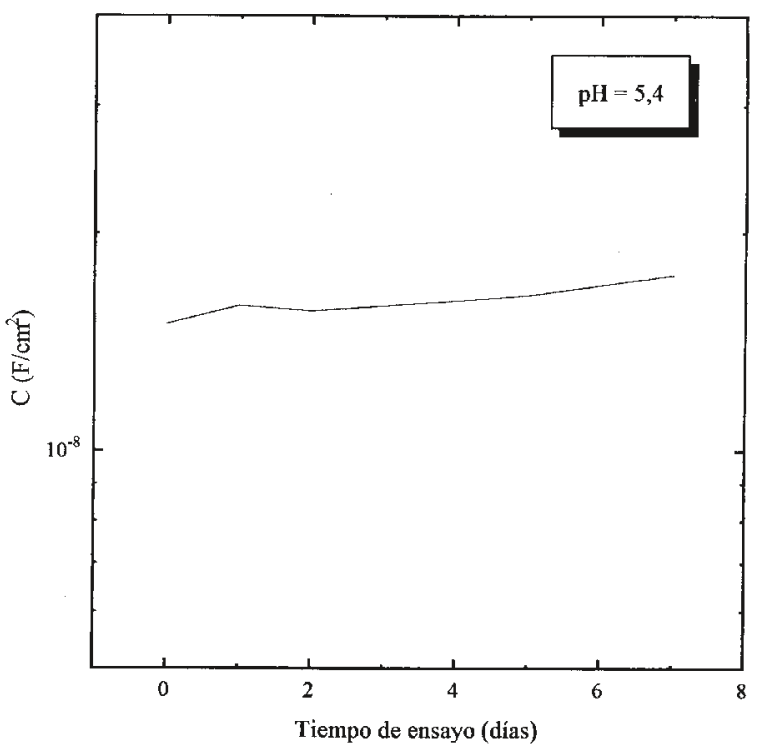

Fig.3.- Variación de la capacidad de la capa de alúmina con el tiempo de ensayo en muestras preoxidadas 3 horas a $1100^{\circ} \mathrm{C}$ en solución de Hank a $\mathrm{pH}=5.4$.

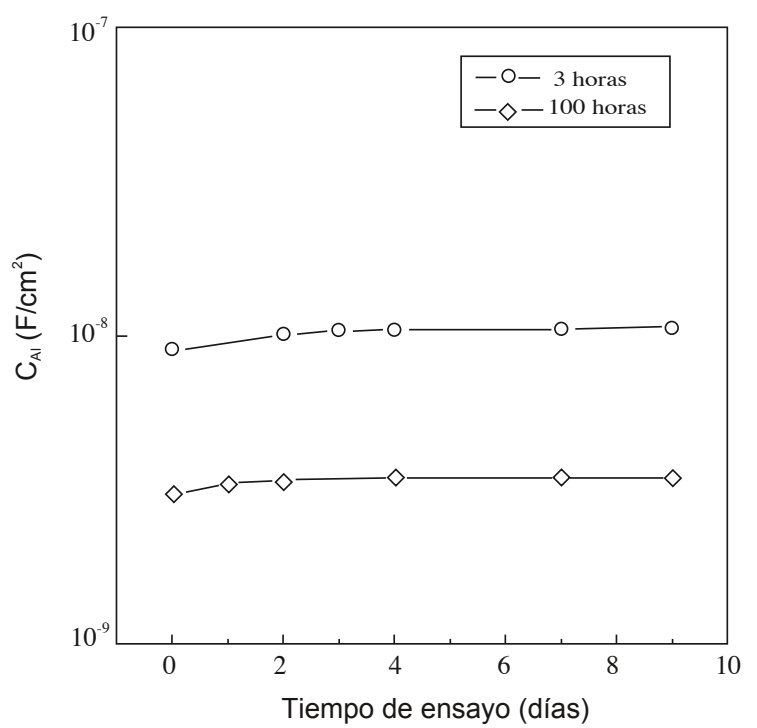

Fig.4.- Variación de la capacidad de la capa de alúmina con el tiempo de ensayo en muestras preoxidadas a $1100^{\circ} \mathrm{C}$ en solución de Hank a $\mathrm{pH}=7.4$.

inerte y estable con independencia del $\mathrm{pH}$ del medio ensayado, neutro o ácido $(\mathrm{pH}=5.4)$. Esta capa actúa como una eficaz barrera frente a la corrosión, aislando en todo momento al sustrato metálico del medio corrosivo expuesto.

Las probetas de MA 956 preoxidadas tambien fueron ensayadas en solución de Hank a $\mathrm{pH}$ ácido $(\mathrm{pH}=5.4)$ y en ausencia de oxígeno, desaireando el medio con nitrógeno, con el fin de simular condiciones de inflamación. Se puede apreciar en la figura 5 como los diagramas de impedancia se superponen, mostrando un mismo comportamiento frente a la corrosión con independencia de que la disolución contenga o no oxígeno. Este comportamiento similar pone de manifiesto, nuevamente que en ningún momento hay conexión entre sustrato metálico y medio corrosivo a traves de los pequeños microdefectos que se encuentren en la capa. 


\section{CONCLUSIONES}

El comportamiento frente a la corrosión de la aleación MA 956 preoxidada ensayada en condiciones desaireadas, y de $\mathrm{pH}$ ácido, $\mathrm{pH}=5.4$, simulando condiciones in vivo diversas como hematoma, infección o inflamación, es excelente, no evidenciándose ningún tipo de disolución de la capa de alúmina, ni conexión entre sustrato metálico y medio corrosivo.

\section{AGRADECIMIENTOS}

Loa autores agradecen la financiación a la Comisión Interministerial de Ciencia y Tecnología (CICYT) del proyecto: MAT95-0249-01 y el haber otorgado una beca de la Comunidad de Madrid con referencia CAM AE 94/95 para M.C. García Alonso.

\section{BIBLIOGRAFIA}

1. D.C. Mears. Metals in Medicine and Surgery. Inter. Met. Rev. 119-55, 1977.

2. J. Black. Biological performance of materials. Vol. 8 of Biomedical engineering and instrumentation. 3, (1981)

3. M. Castro. L'emploi des alliages metalliques en orthopedie. Conf. XL Reunion annuelle de la societé d'orthophedie et de traumatologie. (1970).

4. E. Frank, H. Zitter. Implantes metálicos en cirugía ósea. Ed. JIMS, Barcelona 62-63, (1974).

5. J. Black y col. Metallosis associated with a stable titanium-alloy femoral component in total hip replacement. J. Bone Joint. Surg. Am. 72; 126-130, 1990.

6. M.L. Escudero, J.L. González-Carrasco. In vitro corrosion behaviour of MA 956 superalloy. Biomaterials. 15; 1175-1180, (1994).

7. M.L. Escudero, J.L. González-Carrasco, C. García-Alonso, E. Ramírez. Electrochemical impedance spectroscopy of preoxidized MA 956 superalloy during in vitro experiments. 16; 735-740, (1995).

8. M.L. Escudero, M.F. López, J. Ruiz, M.C. García-Alonso, H. Canahua Comparative study of the corrosion behaviour of MA-956 and conventional metallic biomaterials. 31; 313-317, (1996).

9. M. Pourbaix. Atlas of electrochemical equilibria in aqueous solutions. Houston TX, NACE, (1974).

10. H. Leidhesier, M.W. Kendig. Corrosion.The mechanism of corrosion of polybutadiene-coated steel in aerated sodium choride.32; 69-76, (1976).

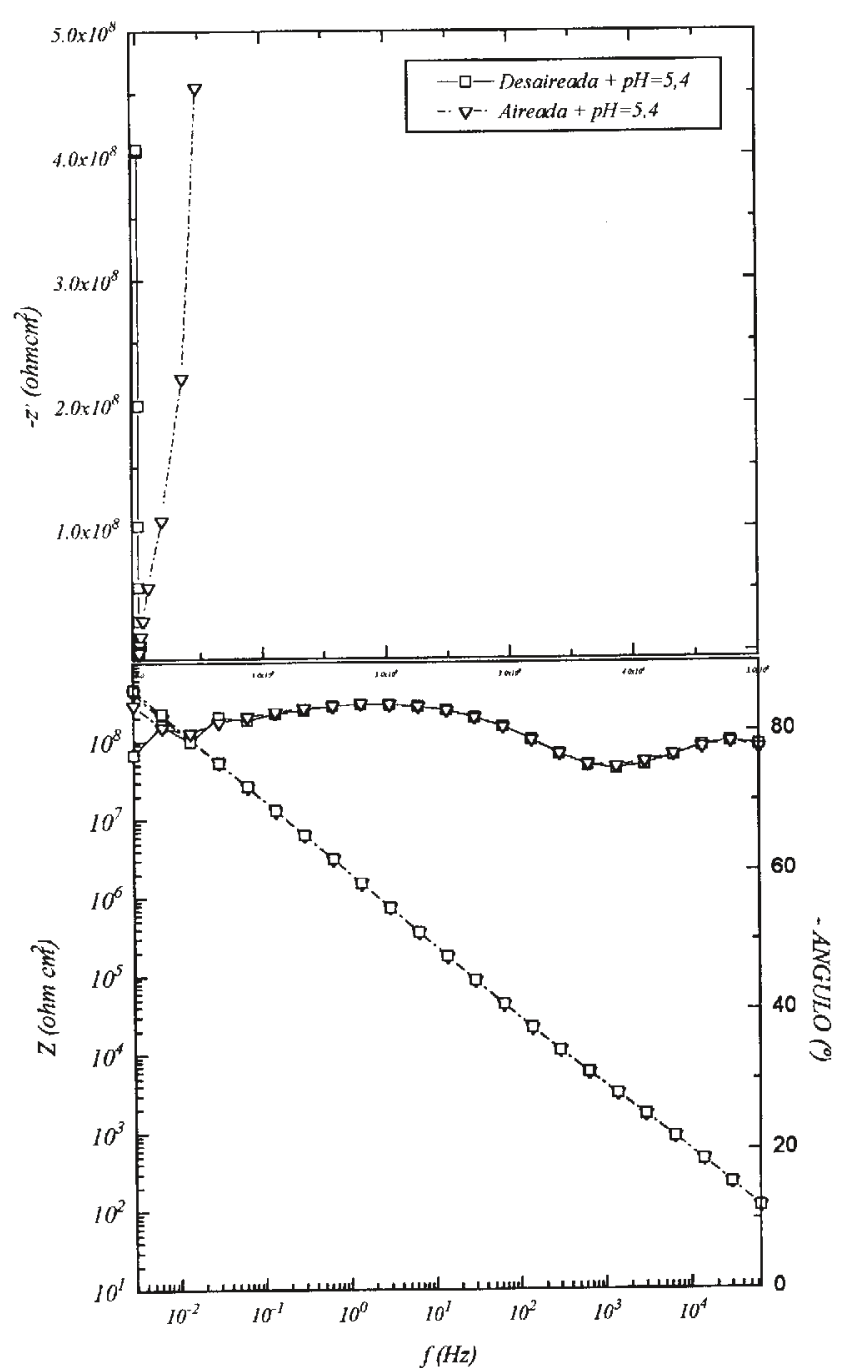

Fig.5.- Diagramas de impedancia de Nyquist y de Bode para muestras preoxidadas 3 horas a $1100^{\circ} \mathrm{C}$ en medio ácido $\mathrm{pH}=5.4$ aireado y desaireado. 